\title{
ADALIMUMAB-INDUCED MULTIPLE SCLEROSIS IN A PATIENT WITH IBD-RELATED ARTHRITIS
}

\author{
M.H. Gharib, H.M. Abdulaziz
}

Hamad Medical Corporation, Medicine/Rheumatology,

TNF blockers have revolutionized the management of immune-mediated inflammatory diseases. Despite their relatively safe profile, an increasing number of reported neurological adverse events suggest a role for TNF inhibitors in the induction of central and peripheral nervous system demyelination.

We report a 21 year-old Qatari gentleman who was on adalimumab for HLA-B27 negative peripheral spondyloarthritis. After being maintained on adalimumab for 2 years, he presented acutely with headache, urinary incontinence and bilateral lower limb numbness that progressed to mid-trunk over 2 days. The neurological examination revealed dysesthesias of lower extremities and trunk up to T4 dermatomes with brisk tendon reflexes. His muscle power and cranial nerves examination were normal.

A diagnosis of multiple sclerosis has been suggested by the typical findings of classical multiple hyper-intense enhancing lesions (figure 1). Our patient has no family history for such diagnosis.
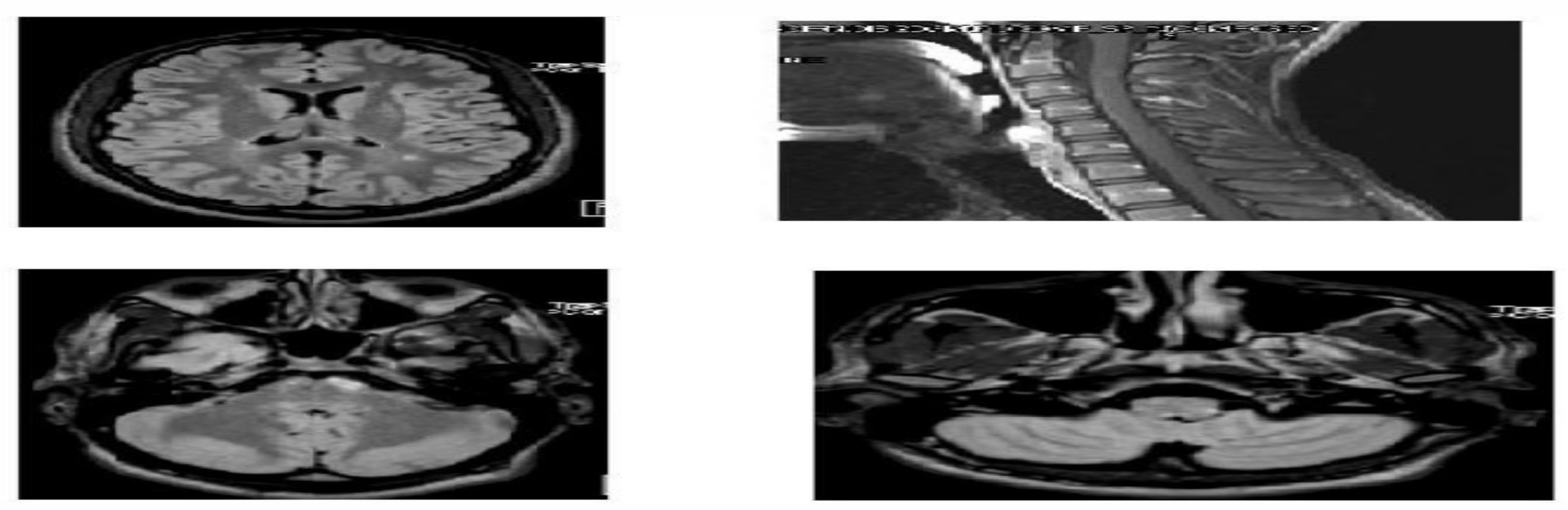

[figure 1: MRI scan showing multiple hyper intense white matter lesions]

Discontinuation of Adalimumab resulted in complete resolution of the neurological symptoms within a month. However, he then developed abdominal pain with loose bloody motions for which lliocolonoscopy and biopsy confirmed the diagnosis of Crohn's disease.

We think that his Gl symptoms were unmasked by the discontinuation of his Adalimumab. Interestingly we found that he has a strong family history of inflammatory bowel disease.

This case illustrates the importance of being vigilant for neurological side effects of anti-TNFa blockers with early discontinuation of the TNF-a blockers and requesting urgent MRI scan. Long term follow up is mandated in such cases to trace relapse rate and clinical outcome. 